\title{
Correction to: On Gaussian covert communication in continuous time
}

\author{
Ligong Wang
}

Correction to: EURASIP J. Wireless Commun. Netw. (2019) 2019:283

https://doi.org/10.1186/s13638-019-1609-8

Following publication of the original article [1], the author flagged that the article had published with an error in one of the equations (Eq. 46), in addition to a few minor formatting errors (concerning spacing and parentheses).

The error regarding the equation was that ' $\mathrm{E}$ ' was missing from the beginning of the equation.

Please see the corrected equation here for reference:

$$
\mathrm{E}\left[\hat{Y}^{2}\right]=a^{2}+\frac{\mathrm{N}_{0}}{2}
$$

The errors have now all been corrected in the published article.

Published online: 30 January 2020

\section{Reference}

\footnotetext{
1. L. Wang. On Gaussian covert communication in continuous time. EURASIP J, Wireless Commun. Netw. 2019, 283 (2019). https://doi.org/10.1186/s13638019-1609-8
}

\footnotetext{
Correspondence: ligong.wang@ensea.fr

The original article can be found online at https://doi.org/10.1186/s13638019-1609-8

ETIS-Université Paris Seine, Université de Cergy-Pontoise, ENSEA, CNRS, Cergy-Pontoise, France
} 